\title{
Novel percutaneous access to the large intestine using a double-balloon endoluminal platform for excision of a large polyp
}

Endoluminal therapies (e.g. tumor excision, hemostasis, closure of intestinal defects) can be complex [1]. There are reports of successful percutaneous access to treat upper gastrointestinal

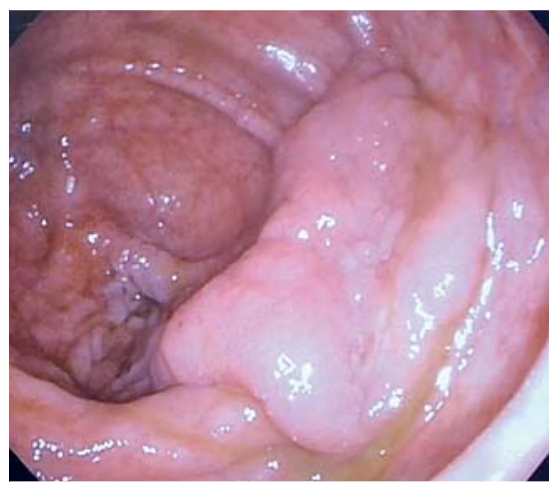

- Fig. 1 Endoscopic view showing a large sessile polyp located at the posterior wall of the cecum.
(GI) lesions [2]; however, percutaneous access has not been established yet in the treatment of lower GI conditions [3]. We here report a novel method of percutaneous access to the colon for excision of a large polyp.

A 72-year-old woman had a large sessile polyp $(60 \times 40 \mathrm{~mm})$ at the posterior wall of the cecum ( $\triangleright$ Fig. 1). A colonoscope (PCF-H180AL; Olympus) equipped with a double-balloon endoluminal interventional platform (DEIP; DiLumen; Lumendi, Westport, Connecticut, USA) ( Fig.2) was advanced to the cecum. The DEIP was used to shorten and stabilize the colon, so facilitating instrument exchange and specimen retrieval $[4,5]$. Using fluoroscopic guidance, we inserted three T-fasteners (Gastrointestinal Anchor Set; Halyard, Alpharetta, Georgia, USA) to secure the colon to the abdominal wall, then inserted 8-Fr and
10- Fr sheaths (AVANTI Sheath Introducer; Cordis, Santa Clara, California, USA) inside the T-fastener triangle. A mini-laparoscopic grasper and electrosurgery (Gimmi, Tuttlingen, Germany) were introduced through vascular sheaths (> Fig. $\mathbf{3}$ ).

Immediately after a submucosal injection (ORISE Gel, Boston Scientific), these tools were used to provide traction and perform mucosal incision and submucosal dissection under endoscopic visualization. The site of bleeding was precisely visualized using one grasper and endoscopic irrigation and was coagulated using the other grasper ( $\triangleright$ Fig. 4 ). The resected specimen was retrieved through the DEIP sheath. Mucosal closure was greatly facilitated by using the two graspers to approximate the two edges of the defect. The vascular sheaths were then withdrawn, and the percutaneous
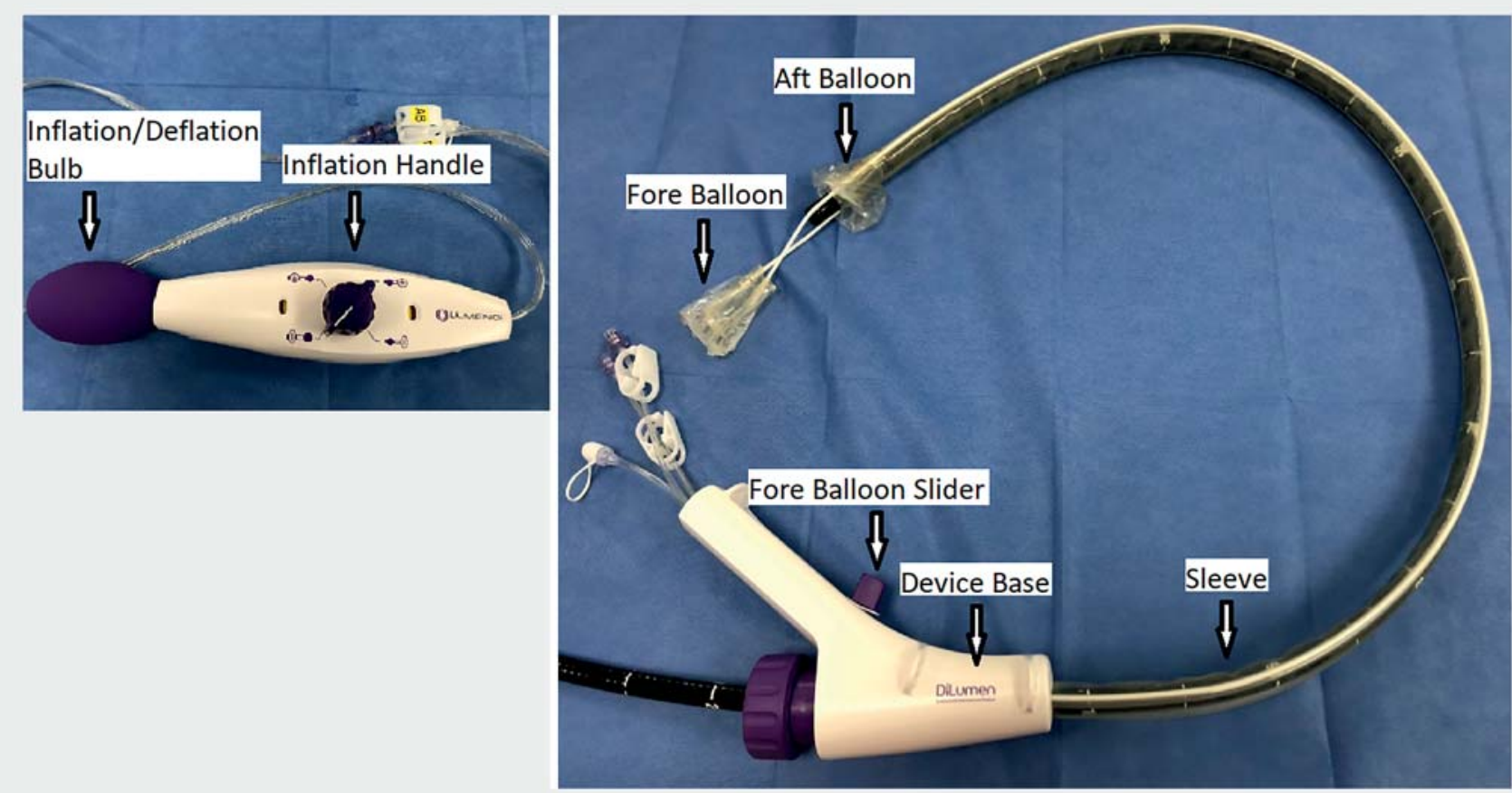

- Fig. 2 The double-balloon endoluminal platform (DiLumen; Lumendi). 

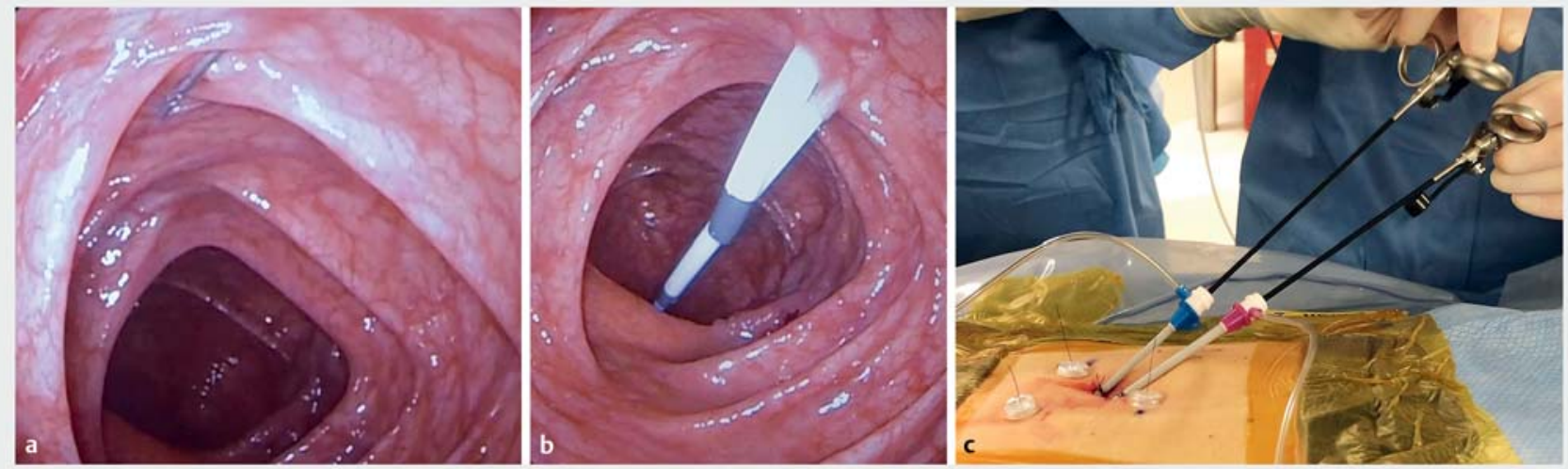

- Fig. 3 Images showing the setup for percutaneous access: a three T-fasteners were inserted into the ascending colon (yellow arrow) to fix it to the abdominal wall in a triangular shape; $\mathbf{b} 10-\mathrm{Fr}$ and $8-\mathrm{Fr}$ vascular sheaths were inserted inside the T-fastener triangle; $\mathbf{c}$ various mini-laparoscopic tools (2.8-mm external diameter) were passed through vascular sheaths.
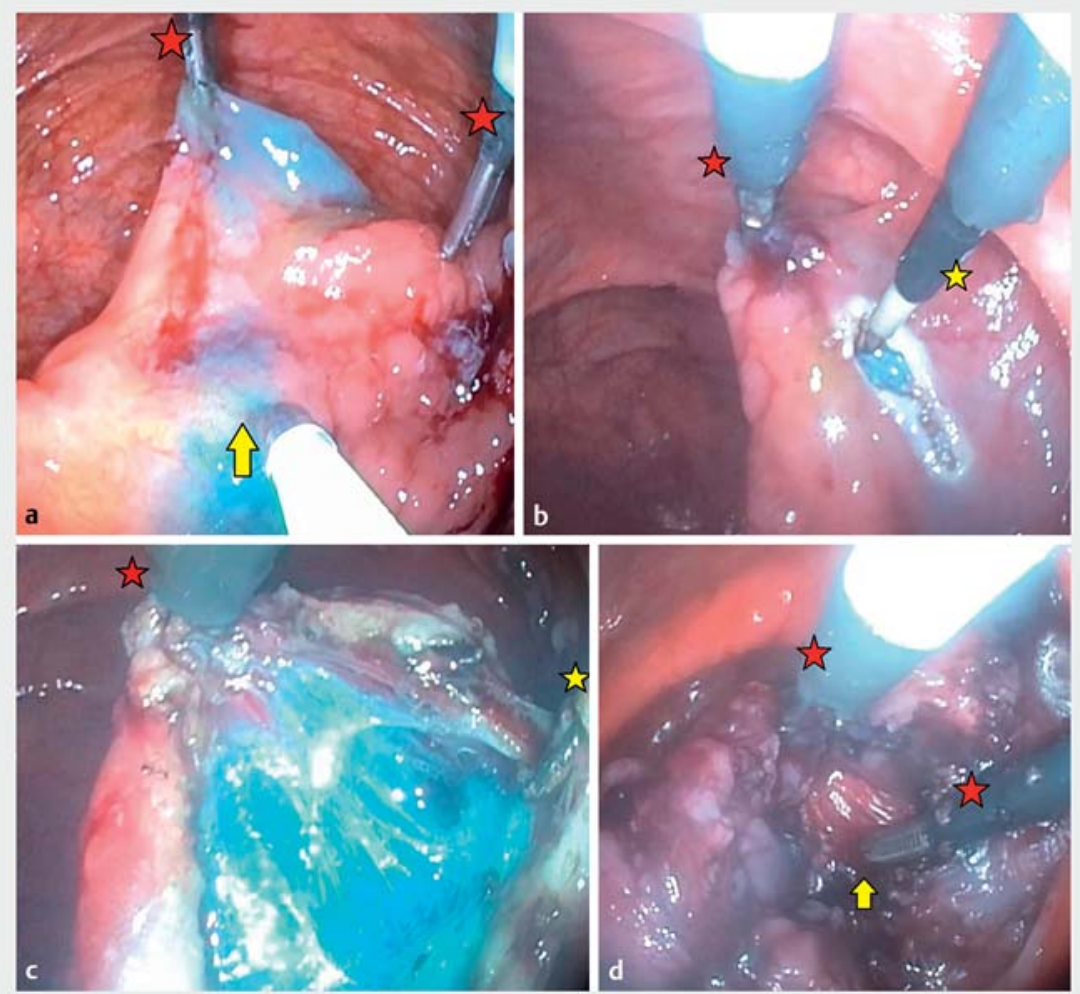

- Fig. 4 Images showing polyp excision using the percutaneous access and laparoscopic tools: a submucosal injection (yellow arrow) facilitated by the laparoscopic graspers (red stars); b mucosal incision using electrosurgery (yellow star); c submucosal dissection; d hemostasis (yellow arrow).

entry points were closed with endoscopic clips. Fluoroscopic evaluation revealed no contrast extravasation from the colon ( $\triangleright$ Fig. $\mathbf{5}$; $\triangleright$ Video $\mathbf{1}$ ).

The patient was discharged the following day, had an uneventful postoperative course, and her wounds healed well
( Fig.6). The final pathological analysis showed a tubular adenoma, with clear margins.

Three other patients have also undergone percutaneous access for other intestinal conditions. All of the procedures were successful, without proce- dure-related complications. This early experience suggests that percutaneous access to the colon may have many clinical applications.

Endoscopy_UCTN_Code_TTT_1AQ_2AD

\section{Competing interests}

The authors declare that they have no conflict of interest.

\section{The authors}

Shinya Urakawa ${ }^{1}$, Daniel Joseph Holzwanger ${ }^{2}$, Teijiro Hirashita ${ }^{1}$, Lea Lowenfeld ${ }^{1}$, Kelly A. Garrett $^{1}$, Bradley B. Pua ${ }^{2}$, Jeffrey W. Milsom ${ }^{1}$

1 Section of Colon and Rectal Surgery, Department of Surgery, Weill Cornell Medicine/New York-Presbyterian Hospital, New York, New York, USA

2 Department of Interventional Radiology, Weill Cornell Medicine/New YorkPresbyterian Hospital, New York, New York, USA

Corresponding author

\section{Jeffrey W. Milsom, MD}

Department of Surgery, Weill Cornell Medicine/New York, Presbyterian Hospital, 525 East 68th Street, K-801, New York, NY 10065, USA

milsomj@gmail.com 

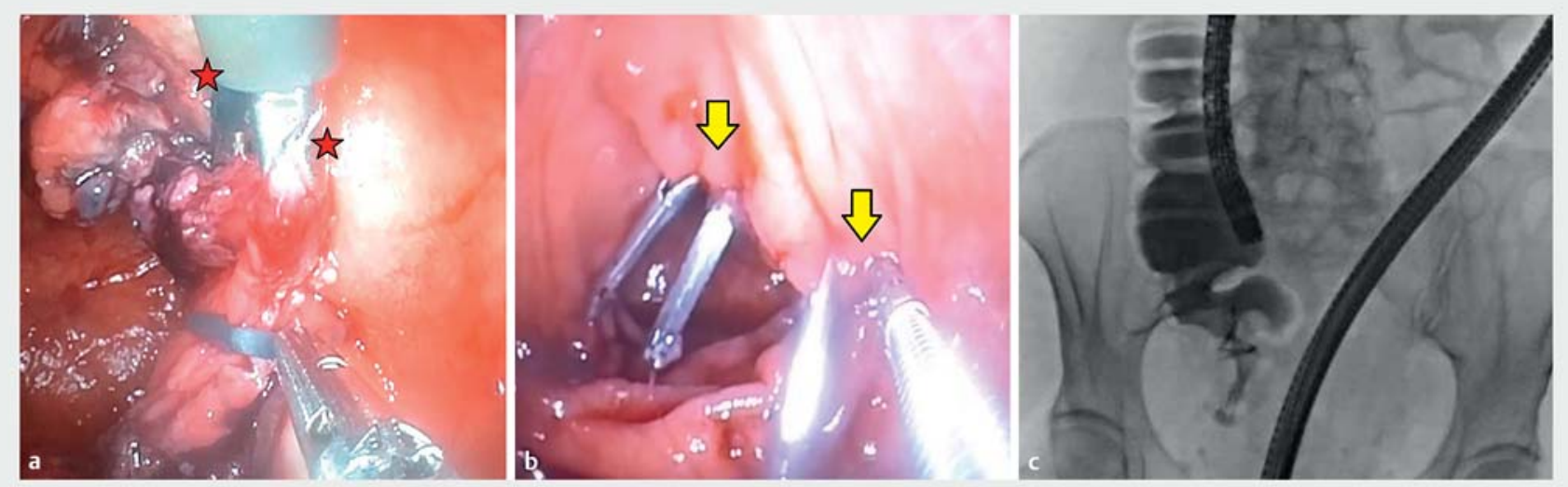

- Fig. 5 Images showing the final stages of the procedure including: a closure of the mucosal defect with endoscopic clips (red stars indicate the laparoscopic graspers); b closure of the percutaneous entry sites with endoscopic clips (yellow arrows); c fluoroscopy to exclude contrast extravasation at the percutaneous entry sites.

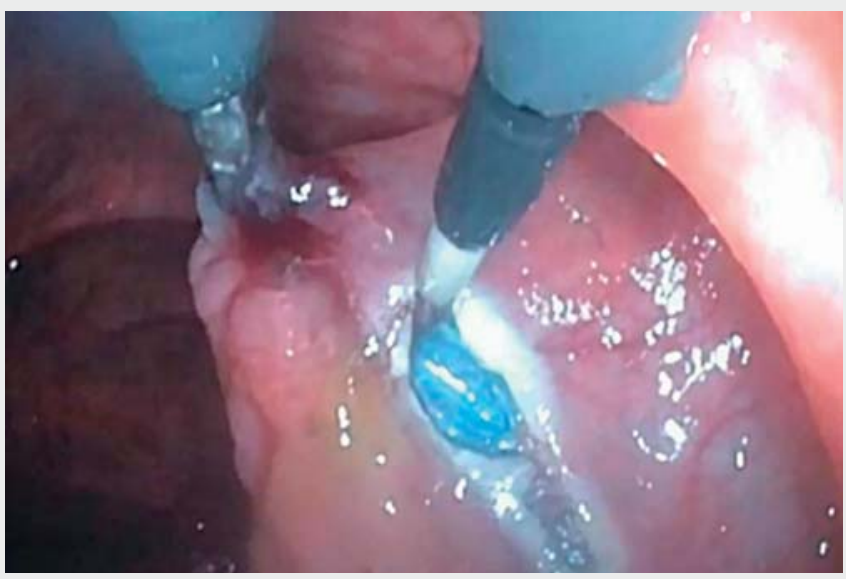

Video 1 Use of the double-balloon endoluminal interventional platform (DEIP) to achieve percutaneous access to the cecum for excision of a large polyp.

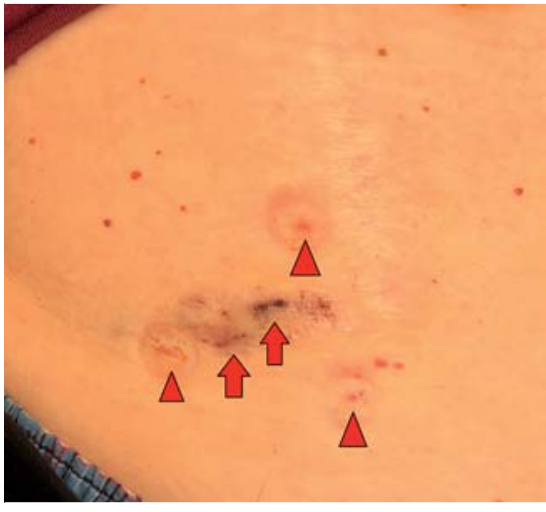

Fig. 6 Photograph showing the right lower abdominal wound 1 week after the procedure with the puncture sites of the T-fasteners (yellow arrowheads) and vascular sheaths (red arrows) indicated.

Bibliography Endoscopic stabilization device evaluation using IDEAL framework: A quality improvement study. Int J Surg 2019; 67: 18-23

[5] Urakawa S, Momose K, Hirashita T et al. Endoscopic submucosal dissection of large polyps in the right colon using an endoscopic snare with a double-balloon endolumenal interventional platform: an ex vivo study in a porcine colorectal model. Surg Endosc 2020. doi:10.1007/s00464-02008100-7
Endoscopy 2022; 54: E331-E333

DOI 10.1055/a-1516-3533

ISSN 0013-726X

published online 19.7.2021

(c) 2021. Thieme. All rights reserved.

Georg Thieme Verlag KG, Rüdigerstraße 14 70469 Stuttgart, Germany 